\title{
Phylogenetic Relationships in Actinidia as Revealed by RAPD Analysis
}

\author{
Hongwen Huang, Zuozhou Li, and Jianqiang Li \\ Wuhan Institute of Botany, The Chinese Academy of Sciences, Wuhan, Hubei 430074, P.R. China \\ Thomas L. Kubisiak \\ U.S. Department of Agriculture Forest Service, Southern Research Station, Southern Institute of Forest \\ Genetics, Saucier, MS 39574 \\ Desmond R. Layne ${ }^{1}$ \\ Department of Horticulture, Clemson University, Clemson, SC 29634-0375
}

\begin{abstract}
ADDITIONAL INDEX WORDs. intrageneric subdivision, geographic groups, kiwifruit
Abstract. Phylogenetic relationships within the Actinidia were investigated using randomly amplified polymorphic DNA (RAPD) markers. DNAs from 40 taxa, including 31 species encompassing all four sections and four series of the traditional subdivisions within the genus, were amplified using 22 preselected 10-mer oligonucleotide primers. A total 204 DNA bands were scored across the 40 taxa, of which $188(92 \%)$ were polymorphic. A wide range of genetic similarity was observed among the taxa $(0.13$ to 0.61$)$. The average similarity between varieties of the same species was 0.54 , and between different species was $\mathbf{0 . 2 8}$, respectively. Although the phylogenetic analysis revealed a clear indication that section Leiocarpae was a monophyletic group, subdivisions of the other three traditional sections were poorly supported. The UPGMA phenogram showed that the majority of the species clustered into geographic subgroups in accordance with their natural distribution (the Yangtzi River, southeastern China, southern China and southwestern China). The intrageneric subdivisions of Actinidia appeared to be difficult, but some subdivisions could be explained by the geographic distribution of the species, particularly for species of Liang's sections of Maculatae and Stellatae. The phylogenetic relationships among several species with previous taxonomic uncertainty are also discussed on the basis of the RAPD data. The results of this study supplement our previous understanding of the Actinidia taxonomy based solely on morphological characters.
\end{abstract}

The genus Actinidia Lindl. belongs to the family Actinidiaceae and comprises 66 species and 118 taxa according to a recent report (Huang et al., 2000). The best-known species are $A$. deliciosa (A. Chev.) C.F. Liang et A.R. Ferguson and A. chinensis Planch., from which most commercial kiwifruit varieties have been developed. Since economic potential of A. deliciosa was exploited following a single seed introduction into New Zealand from China in 1904 and the first commercial orchard was established in New Zealand in 1930 (Ferguson and Bollard, 1990), an international kiwifruit industry of more than 100,000 hectares with an annual production of one million tons has been developed since the early 1970s (Huang and Ferguson, 2001). A rapid expansion of the industry has brought about an increased interest in broadening the genetic base of the breeding programs, and further exploitation of related species has rekindled an interest in botanists and horticulturists to try to better understand the phylogenetic relationships and taxonomic hierarchy within the genus (Ferguson, 1990a; Li, 1952; Liang, 1984), which is prerequisite to formulating the appropriate germplasm accession management strategy in the kiwifruit repositories.

Actinidia has a remarkably wide geographic distribution in eastern Asia, extending from the equator (tropics) to cold temperate regions as far north as $50^{\circ}$ latitude (Ferguson, 1990a; Liang,

Received for publication 12 Dec. 2001. Accepted for publication 7 June 2002. This research was partially supported by State Key Basic Research and Development Plan of China (G2000046806), NSF of China (30070082), the European Union (INCO-DC IC18CT970183) and the Chinese Academy of Sciences. We are grateful to A.R. Ferguson, HortResearch, New Zealand and R. Testolin, University of Udine, Italy for critically reviewing the manuscript and providing valuable comments.

${ }^{1}$ Assistant professor of pomology and extension fruit specialist; corresponding author; e-maildlayne@clemson.edu.
1983). In general, however, the native distribution of most taxa of Actinidia is centered around the mountains and hills of south central and southeast China with the QinLing mountains forming a northern boundary and the HengDuan mountains forming a western boundary. In addition to four species native to neighboring countries [A. strigosa Hook. f. et Thoms. found in Nepal, $A$. petelotii Diels in Vietnam, A. hypoleuca Nakai and A. rufa (Sieb. et Zucc.) Planch. ex Miq. in Japan], 62 species, about 45 varieties, and seven forms have been found in China (Cui, 1993; Ferguson, 1990a; Liang, 1983). All members of Actinidia are dioecious perennial climbing vines characterized by obligate outcrossing. The variation in morphological characters, chemical contents, ploidy levels, isozyme markers, and DNA markers is tremendous among taxa within the genus, as recently discussed by Huang et al. (2000). In particular, the variation in ploidy level includes diploids $(2 \mathrm{n}=58)$, tetraploids $(2 \mathrm{n}=116)$, hexaploids $(2 \mathrm{n}=174)$, and occasional octaploids $(2 \mathrm{n}=232)$, forming a reticulated intraspecific and interspecific structure within the genus (He et al., 1998; Huang et al., 2000; McNeilage and Considine, 1989; Xiong and Huang, 1988; Yan et al., 1994, 1997).

The taxonomy of Actinidia has remained equivocal since Lindley erected the name Actinidia in 1836. An early taxonomic treatment by Gilg (1893) split eight species into two groups based on types of inflorescence (solitary and cyme). In the first systematic revision, Dunn (1911) recognized 24 species and established four sections Vestitae, Maculatae, Ampulliferae and Leiocarpae, based on the degree of pubescence, shape of ovary, and presence or absence of lenticels on the fruit surface. Li (1952), by emphasizing the structure of leaf hairs and by eliminating the ambiguous character of ovary shape in the second revision, divided the section Vestitae into two sections Stellatae and Strigosa, merged the section Ampulliferae into section Leiocarpae, and retained 
Table 1. List of the Actinidia taxa sampled in the present study and their original distribution

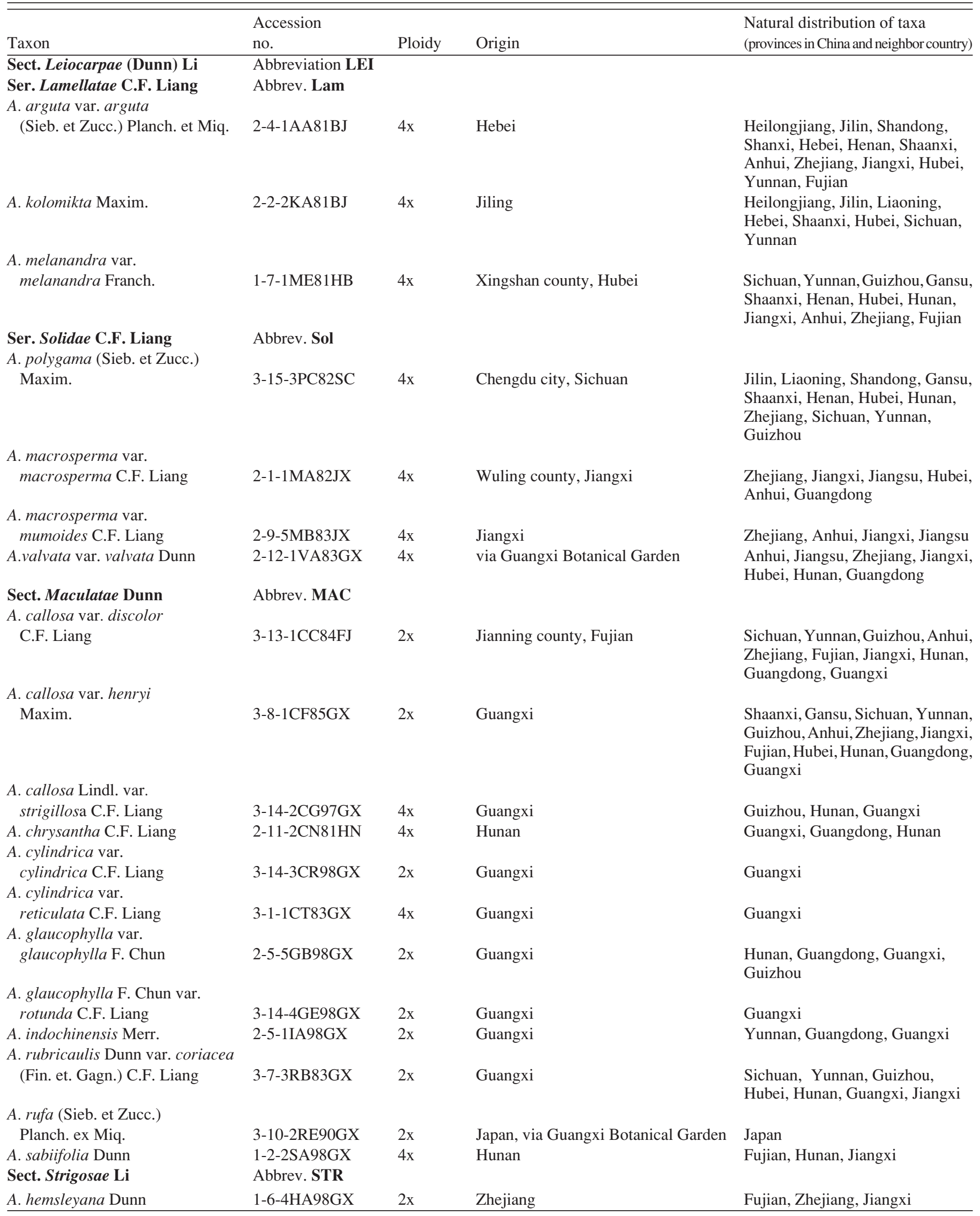


Table 1. Continued.

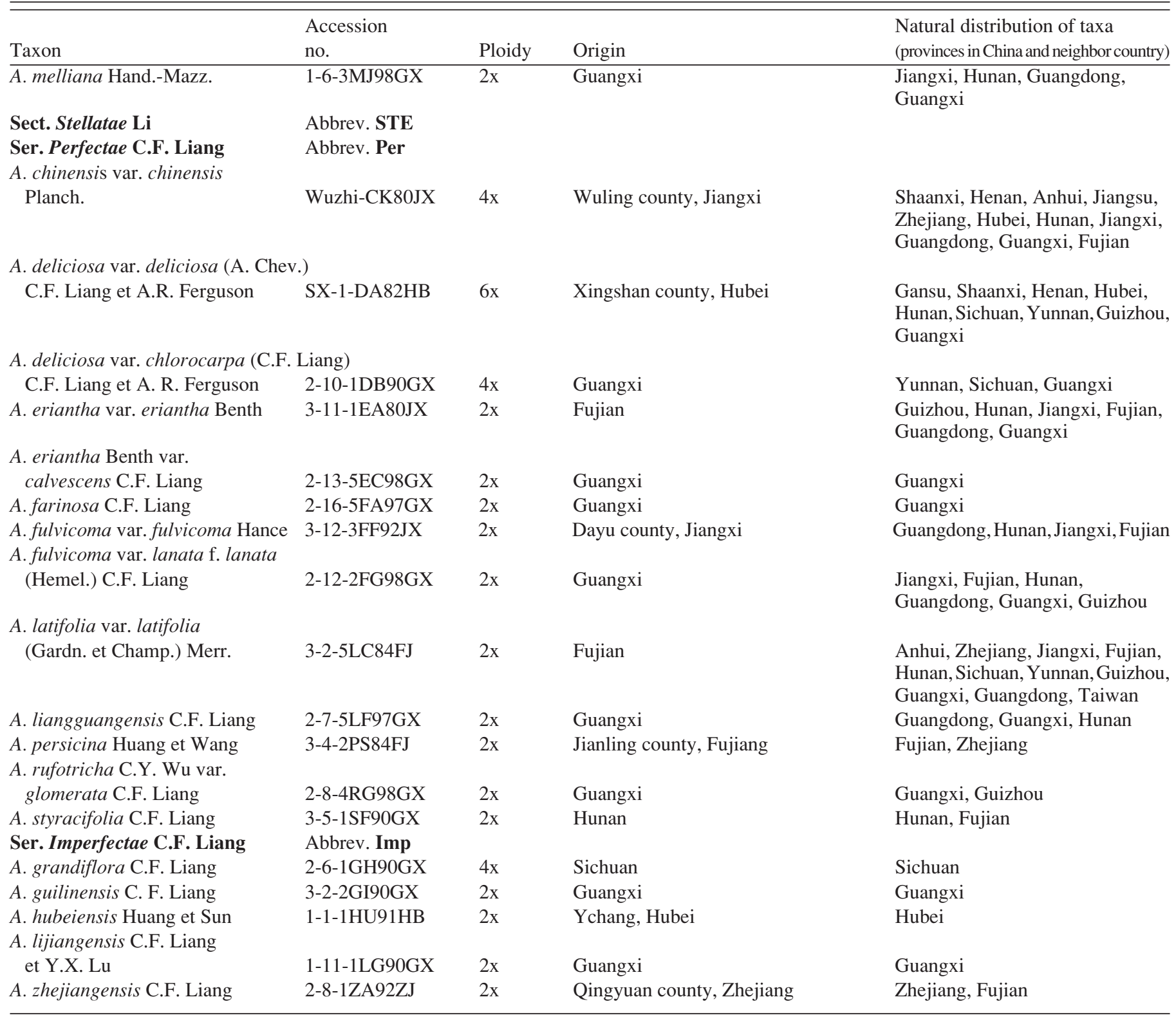

the section Maculatae. Thirty-five species and 14 varieties were described in his revision. The most recent revision by Liang (1984) retained Li's division of the four sections but with a modification of further subdividing two series Lamellatae and Solidae within section Leiocarpae and two series Perfectae and Imperfectae within section Stellatae by taking into account stem pith structure and stellate hairs, respectively, in each section. He significantly increased the number of taxa to a total of 51 species, 35 varieties and six forms (Liang, 1984). Since then, there have been many new species published (Huang and Wang, 1995; Jiang, 1995; Shi et al., 1994; Sun and Huang, 1994) and the intrageneric subdivisions have again come into question. Phylogenetic analyses based on 20 to 50 morphological characters as well as microstructures of leaf trichomes resolved all species with a smooth fruit skin as a monophyletic group (sectionLeiocarpae), but subdivision of the other sections was ambiguous (He et al., 2000; Huang et al., 1999; Li et al., 2000). Phylogenetic relationships of a limited number of taxa as revealed by allozyme and cpDNA markers were also unable to clearly subdivide the other three sections (Cipriani et al., 1998; Testolin and Ferguson, 1997). In general, the taxonomy of the Actinidia based on morphological characters is not clearly resolved and the phylogenetic relationships within the genus are difficult to assess because the boundary between intra- and interspecific classification is blurred by the extensive variation of the morphological characters, and the fact that the various transitional forms existing between taxa have probably resulted from natural hybridization between species with sympatric distributions (Ferguson, 1990a). Little is known about the speciation process in Actinidia, although the genus has been speculated to be undergoing a fast speciation in central-southwest China, which is well known for its diverse topography. This region has been considered the center of diversity of the genus and is where most of the new species of Actinidia are being found (Liang, 1983; Cui, 1993).

A national germplasm repository and breeding program for Actinidia was established in 1978 at the Wuhan Institute of Botany (WIB), in Wuhan, Hubei, People's Republic of China. The long-term goal of the program is to develop a comprehensive 
conservation ex situ repository of all currently known Actinidia species and a genetically rich germplasm collection for further development of superior kiwifruit cultivars. A refined understanding of species boundaries and relationships are of great practical importance for the curator to formulate collecting priorities and sampling strategies and for breeders to use the germplasm resources within the Actinidia genus. The objectives of this study were to 1) examine intrageneric subdivisions in Actinidia for providing accurate information to assist germplasm management of taxonomic entries and accessions in the repository; 2) evaluate the phylogenetic relationships among Actinidia species to aid parent selection in currently ongoing interspecific breeding programs; and 3) compare these results with information on the natural distribution of the various species for future expedition plans to be designed and to enhance collecting efficiency.

\section{Materials and Methods}

Plant materials. All plant materials were collected from the germplasm repository for Actinidia at the WIB. Forty taxa of 31 species, encompassing all four sections and four series of the traditional subdivisions within the genus, were investigated in the present study (see Table 1). Where available, three to five plants of each taxon were included for analysis.

DNA EXTRACTION, PCR AMPLIFICATION. Total nucleic acids were isolated from about $2 \mathrm{~g}$ of fresh leaf tissue using a modification of the cetyltrimethylammonium bromide (CTAB)-based procedure outlined by Wagner et al. (1987). The RNA component of these individual extracts was removed by incubation in the presence of RNase A as described by Ausubel et al. (1987). Oligonucleotide 10-base primers were obtained from Operon Technologies Inc. (Alameda, Calif.). DNA amplification was based on the protocol reported by Williams et al. (1990). The reaction consisted of the following in $24 \mu \mathrm{L}$ total volume: $6.25 \mathrm{ng}$ template DNA, $1 \mu \mathrm{L}$ primer DNA (5 $\mu \mathrm{m}$ stock), $3.6 \mu \mathrm{L}$ dNTPs (1 mu stock), $2.4 \mu \mathrm{L} 10 \times$ Taq DNA polymerase reaction buffer (500 mм KCl, 100 mм Tris-HCl, 1.0\% Triton X-100, $15 \mathrm{~mm} \mathrm{MgCl}_{2}$ ), and 2.0 U Taq DNA polymerase. Reactions were loaded in flexible microtitre plates and overlaid with $25 \mu \mathrm{L}$ of mineral oil. Microtitre plates were placed in preheated $\left(85^{\circ} \mathrm{C}\right) \mathrm{MJ}$ Research PTC-100 programmable temperature cyclers (Watertown, Mass.) and covered with mylar film. The DNA samples were immediately amplified using the following thermal profile: $5 \mathrm{~s}$ at $95^{\circ} \mathrm{C}$; $1 \mathrm{~min} 55 \mathrm{~s}$ at $92^{\circ} \mathrm{C}$; followed by 45 cycles of $5 \mathrm{~s}$ at $95^{\circ} \mathrm{C}, 55 \mathrm{~s}$ at $92^{\circ} \mathrm{C}, 1 \mathrm{~min}$ at $35^{\circ} \mathrm{C}$, and $2 \mathrm{~min}$ at $72^{\circ} \mathrm{C}$; followed by $7 \mathrm{~min}$ at 72 ${ }^{\circ} \mathrm{C}$. The reactions ended with an indefinite hold at $4{ }^{\circ} \mathrm{C}$. Amplification products were electrophoresed in $2 \%$ agarose gels and TAE buffer ( $40 \mathrm{~mm}$ Tris base, $20 \mathrm{~mm}$ sodium acetate, $2.0 \mathrm{~mm}$ EDTA, glacial acetic acid to $\mathrm{pH} 7.2$ ) for about $3.5 \mathrm{~h}$ at $3 \mathrm{~V} \cdot \mathrm{cm}^{-1}$ $(150 \mathrm{~V})$. A total of $3.0 \mu \mathrm{L}$ loading buffer $(10 \times \mathrm{TAE}, 50 \%$ glycerol, and $0.25 \%$ bromophenol blue) was added to each reaction prior to electrophoresis. After electrophoresis, the gels were stained with ethidium bromide $\left(0.4 \mu \mathrm{g} \cdot \mathrm{mL}^{-1}\right)$ for $45 \mathrm{~min}$, washed in distilled water for $1.0 \mathrm{~h}$, and photographed under ultraviolet light using a Polaroid MP-4 camera and Polaroid 667 instant film (Cambridge, Mass.).

DATA ANALYSIS. One hundred and forty-four RAPD primers (Operon Technologies, kits A-G, J and U 01-10) were used for initial screening against eight randomly chosen species to identify RAPD markers. Each sample was amplified at least two times to verify reproducibility. Twenty-two primers that amplified a total of 188 reproducible polymorphic bands were then selected and used in the study. Of the 188 polymorphic markers, 156 markers that showed no polymorphism within taxa were then identified and chosen for the Cluster analysis to reveal phylogenetic relationships between the taxa, and the remaining 32 markers were discarded to avoid intrataxon variation that might confound the analysis of inter-taxa relationships. RAPD markers were designated by the manufacturer primer code corresponding to the 10-mer oligonucleotide primer responsible for their amplification, followed by a four digit number indicating the product size in base pairs (Table 2). RAPD marker phenotypes were

Table 2. The primers used and sizes of 156 intertaxa polymorphic DNA fragments in Actinidia

\begin{tabular}{|c|c|}
\hline Primer code & Size of amplified polymorphic DNA fragments (bp) \\
\hline OPA-07 & $1600,1300,1159,1126,1093,1000,900,805,700,650,514,448,400,350,300,280$ \\
\hline OPA-11 & $1680,1250,1200,1093,1000,850,820,805,640,550,448$ \\
\hline OPA-17 & $1450,1159,1020,805,780,550$ \\
\hline OPC-04 & $1250,1159,900,805,750,730,550,514$ \\
\hline OPD-03 & $1800,1690,950,700,514,490$ \\
\hline OPE-17 & $1250,730,650$ \\
\hline OPE-20 & $1450,1300,1000,680,500,400$ \\
\hline OPF-16 & $1500,1300,1000,650,550,530,339$ \\
\hline OPF-17 & 1700,700 \\
\hline OPF-20 & $1300,1000,550,468,339$ \\
\hline OPG-04 & $1800,1500,1159,1093,1000,820,650,550,455$ \\
\hline OPG-18 & $1700,1550,1300,1200,1093,900,850,700,514,280$ \\
\hline OPJ-07 & $1000,900,650,600,450$ \\
\hline OPU-02 & $1600,900,805$ \\
\hline OPU-06 & $1350,1250,1050,750,700,500$ \\
\hline
\end{tabular}


scored as 1 (band present) or 0 (band absent), respectively. NTSYS-pc (v1.8) was used to compute Jaccard's coefficients of similarity and to construct a phenogram using the unweighted pair group method with arithmetic averages (UPGMA) (Rohlf, 1994).

\section{Results}

RAPD VARIATIONS AND GENETIC SIMILARITY AMONG Actinidia TAXA. A total 204 DNA bands, amplified by 22 different 10 -mer oligonucleotide primers, were scored across the samples. An average of 9.3 DNA bands were amplified per sample/primer combination. Primer OPF-17 amplified as few as two bands, whereas primer OPA-07 amplified as many as 18 bands. The approximate size of the amplified fragments ranged from 280 to $2140 \mathrm{bp}$ (Table 2). Of the 204 DNA bands scored, 188 (92\%) were polymorphic. Across all samples and primers, an average of 8.6 polymorphic bands were amplified per sample/primer combination.

A wide range of genetic similarity was observed among the taxa. The highest genetic similarity $(0.61)$ was observed between A. persicina and A. zhejiangensis, while the lowest genetic similarity (0.13) was between the species A. fulvicoma var. lanata and $A$. sabiifolia. In general, the average similarity between varieties of the same species was 0.54 , and between different species was 0.28 , respectively (data not shown).

Phylogenetic analysis. The phenogram generated by UPGMA clustering analysis revealed two notable observations:

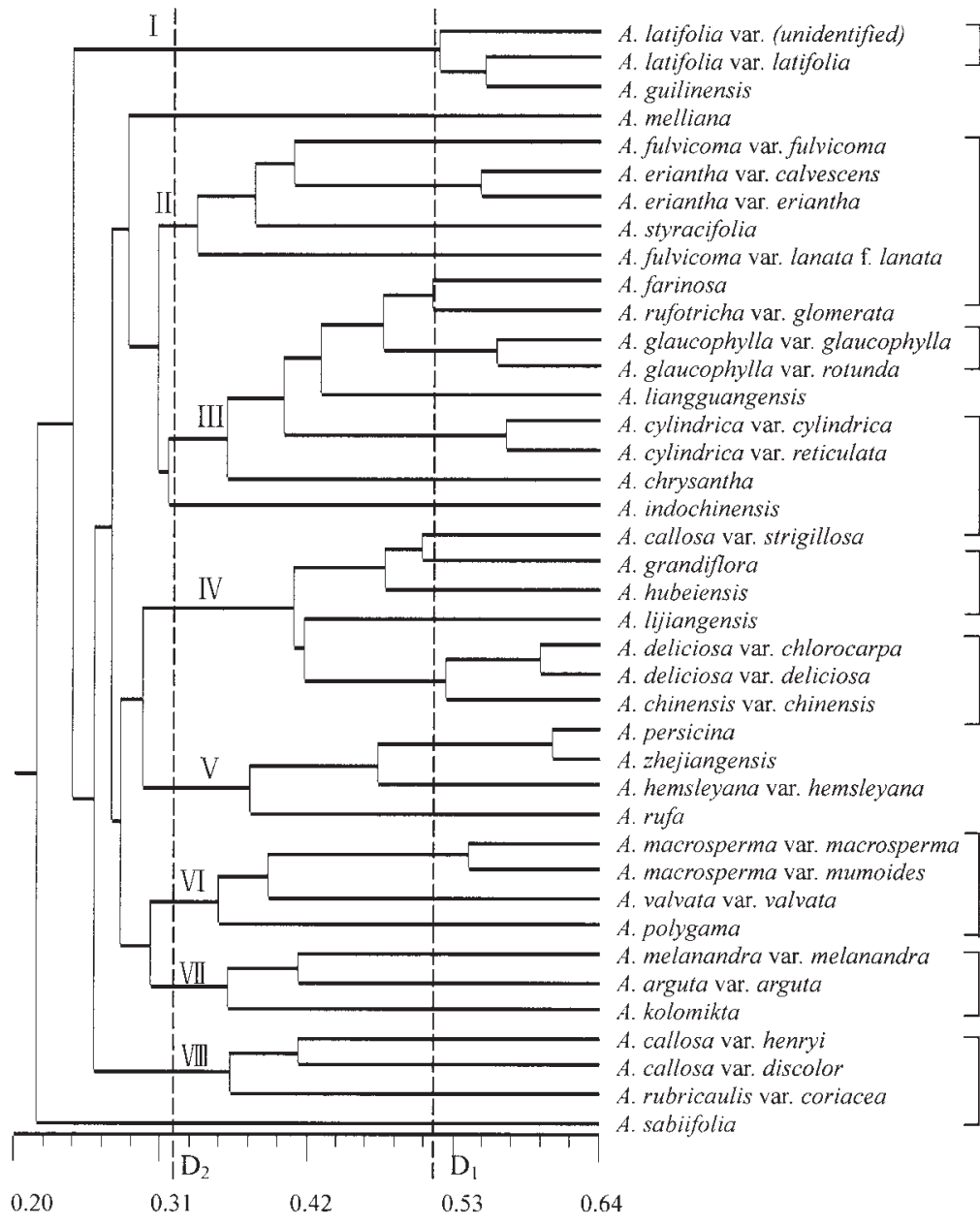

1) different varieties within a species tended to cluster closely with one another; and 2) species within section Leiocarpae formed a distinct cluster, supporting the traditional grouping of all the species with smooth skinned fruit as a monophyletic group, but subdivisions within the other three traditional sections were poorly supported (Fig. 1).

To examine genetic relationships among the taxa, two cut-off points $\left(\mathrm{D}_{1}=0.52, \mathrm{D}_{2}=0.32\right)$ were determined according to $\mathrm{Xu}$ and Li's (1983) method of grouping taxa at different genetic similarity levels (Fig. 1). In general, these two cut-off points showed the separation of species and groups, respectively. At the 0.52 cutoff point, most of the species were separated with only a few exceptions. The genetic similarity between A. latifolia and A. guilinensis was more suggestive of a varietal-level relationship than a species-level relationship. This is consistent with their morphological similarity and the fact that A. guilinensis was once misidentified as a unique genotype of A. latifolia (Liang, 1988). Similarly, A. zhejiangensis and A. persicina formed a close cluster and had similarity measures more suggestive of a varietallevel relationship than a species-level relationship. A fairly high level of genetic similarity was also observed between the two kiwifruit species, A. deliciosa and A. chinensis, from which all of the commercial cultivars have been developed. On the other hand, an unexpectedly low genetic similarity was observed among three varieties of $A$. callosa and between two varieties of $A$. fulvicoma, suggesting that a high degree of genetic variation probably exists within the species. The varieties of $A$. callos $a$ had rather low levels of genetic similarity among one another, var. henryi and var. discolor were clustered at a lower genetic similarity than that suggestive STE - Per of a species-level relationship, while var. STE - Imp strigillosa formed a cluster with A. grandiSTR flora. Two varieties of A. fulvicoma appear to be distantly related to each other.

The 0.32 cutoff point grouped most taxa STE - Per into eight distinct clusters that appear to reflect the geographic distribution of the species, while three remaining taxa $A$. melliana, A.indochinenesis and A. sabiifolia were revealed as individual species (Fig. 1).

MAC STE - Per MAC Cluster I consisted of A. latifolia and A. guilinensis. If $A$. guilinensis were treated as a variety of A. latifolia, this group could be a single species. Cluster II included $A$. STE - Imp fulvicoma, A. eriantha and A. styracifolia.
A.fulvicoma was considered by Li (1952) to be closely related to A. eriantha. The natural STE - Per distribution of the group is centered in Fujian and Guangdong in southeastern China and STE - Imp extends to Guangxi, Guizhou in southwestSTR ern China. Cluster III comprised A. farinosa, MAC A. rufotricha, A. glaucophylla, A. liangLEI - Sol guangensis, A. cylindrica and A. chrysantha. These six species have overlapping distri-

LEI - Lam Fig. 1. UPGMA phenogram based on the similarity (Jaccard's coefficient) matrix calculated from RAPD data of 40 Actinidia taxa. The dotted lines indicate the two cut-off points, $\mathrm{D}_{1}=0.52$ and $\mathrm{D}_{2}=0.32$. The traditional subdivisions are labeled as abbreviation listed in Table 1. Numbers on the phenogram label the seven major clusters. Cophenetic correlation coefficient is 0.76 . 
butions centering in Guangxi and Guizhou in southwest China and spreading over Guangdong and Hunan in southern China. Cluster IV was the kiwifruit complex of $A$. deliciosa and $A$. chinensis plus A. lijiangensis, A. hubeiensis A. grandiflora, and A. callosa var. strigillosa. The natural distribution of the group is centered in Hubei and spreads over the middle reach of the Yangtzi river in central China, except for A. lijiangensis that is primarily located in Guangxi. However, the genetic similarity between A. lijiangensis and other species of the group was reflected well in the phenogram. Cluster $\mathrm{V}$ consisted of $A$. persicina, A. zhejiangensis, A. hemsleyana, and A. rufa. Species in this group are distributed primarily in Zhejiang, Fujian and Jiangxi in eastern China, except for $A$. rufa, which is native to Japan. Cluster VI was the monophyletic group represented by species only in series Solidae section Leiocarpae, while cluster VII was the monophyletic group consisting of only species in the series Lamellatae section Leiocarpae. These two clusters (VI and VII) further formed a larger group reflecting the genetic relationship of section Leiocarpae in accordance with the traditional taxonomy. All these species are naturally distributed in northern China. Cluster VIII included two varieties of A. callosa, var. henryi and var. discolor and A. rubricaulis var. coriacea, suggesting a genetic affinity between $A$. callosa and $A$. rubricaulis var. coriacea. In fact, $A$. rubricaulis var. coriacea was once treated as a variety belonging to $A$. callosa. It was later revised as an independent species A. coriacea (Dunn, 1911; Li, 1952), but in 1984, Liang reclassified it as variety belonging to A. rubricaulis.

\section{Discussion}

Intragenetic SUbDIVISIONS Within Actinidia. The current intrageneric subdivisions within Actinidia have been challenged by several recent investigations (Cipriani et al., 1998; He et al., 2000; Huang et al., 1999; Li et al., 2000; Testolin and Ferguson, 1997; Webby et al., 1994). Two suggestions for a new revision have been proposed. Based on cluster analysis of 50 morphological characters, Huang et al. (1999) proposed a modified subdivision of the genus into three sections: Leiocarpae retaining all species with smooth skinned fruit, Maculatae including the species with spotted fruit and, Vestitae comprising species with leaf hairs by a further dividing into two series, Stellatae for species with stellate leaf hairs and Strigosae for species with simple and/or coarse leaf hairs. Li et al. (2000) suggested a subdivision of two subgenera: Leiocarpae and Maculatae, based on cladistic analysis of 22 morphological characters. However, neither proposal solves the problem that Maculatae is very heterogeneous and contains various leaf hair types and various degrees of spotted fruits, making it particularly difficult to delimit species in the Maculatae and Vestitae. Analyses including a number of different metrics such as general morphology, leaf flavonoid content, isozymes, and cpDNA have all provided evidence for grouping species with smooth skinned fruit as a monophyletic section, but subdivision of the other three traditional sections were ambiguous (Cipriani et al., 1998; He et al., 2000; Huang et al., 1999; Li et al., 2000; Testolin and Ferguson, 1997; Webby et al., 1994). Phylogenetic analysis using RAPDs provides further evidence to support the section Leiocarpae as a monophyletic group, but similar to other metrics studied to date, it does not provide any convincing evidence for subdividing the other sections. The UPGMA phenogram clearly showed the section Maculatae as a polyphyletic group with most members either standing out as single species or clustered with species of the section Stellatae or section Strigosae (Fig. 1). A similar situation was also observed in Liang's Stellatae. Liang's section Strigosae has been speculated to be an artificial group because of a lack of morphological characters common to the species within it, and because the species all have scattered geographic distribution patterns (Liang, 1983). Two typical species of the Strigosae section, A. melliana and A. hemsleyana, were examined in this study and found not to be closely related. The UPGMA phenogram showed that many species were clustered into geographic subgroups in accordance with their known distributions. About $60 \%$ of the species within the genus Actinidia are found in southwestern China, where the complicated topography has created a myriad of microclimates influenced by the mountain ranges in the region (Cui, 1993). A geographic distribution pattern associated with speciation has already been suggested (Liang, 1983). The more varied the geographic environments are within a region, the more diverse are the genotypes and taxa (Zao and Liu, 1996). Based on this study and previous data on reticulated ploidy structure (Ferguson, 1990a; Huang et al., 2000), the frequent occurrence of natural hybridization and cross compatibility (Ferguson, 1990b; Wang and Huang unpublished data) and cpDNA analysis (Cipriani et al., 1998), it is reasonable to hypothesize that hybridization is functioning to produce a reticulate evolutionary structure within the Actinidia. Liang's section Leiocarpae is most likely an ancestral group. The species $A$. polygama and A. kolomikta could be considered progenitor species. A. arguta, A. melanandra and A. macrosperma could be more recent derivative species that are still undergoing rapid speciation as the largest numbers of varieties are found within these species (Liang, 1983). It is possible that the spotted fruit species in Liang's section Maculatae could be in transition from a progenitor species with smooth skinned fruit (section Leiocarpae) to a pubescent species (most species of section Stellatae have more or less a gradation of hairs on the fruit). Meanwhile, overlapped distributions, mutation and natural hybridization may have created a geographically oriented polyphyletic origin to the groups creating an apparent mixed structure to many of the species including Liang's sections Stellatae and Strigosae. For this reason, a new revision of intrageneric subdivisions of Actinidia might need to take into account the geographic distribution of the particular species, especially for species in Liang's Maculatae and Stellatae.

Phylogenetic Relationships between the species. The genetic relationships between species revealed by cluster analysis of RAPDs are generally consistent with those described or discussed in traditional taxonomy, but some interesting results and new observations relevant to taxonomy should be noted.

With respect to the affinity between $A$. latifolia and $A$. guilinensis, a close relationship between the species was revealed suggesting a possible varietal-level relationship rather than a species-level relationship. Further, A. latifolia appears to harbor a great deal of variation as determined by the low levels of similarity (Fig. 1). This result supports the morphological observations that $A$. guilinensis is quite similar to A. latifolia (Cui, 1993; Liang, 1988). A. latifolia has a wide geographic range from southeastern to southwestern China, while A. guilinensis is narrowly endemic to Guangxi province (Li, 1952; Liang, 1983, 1988). A. guilinensis is speculated to have originated from natural hybridization with $A$. latifolia as a parent and may be undergoing rapid speciation (Cui, 1993; Liang, 1988). A. guilinensis was classified as a species mostly based on one morphological character that its mature leaf becomes glabrous (Liang, 1988). In fact, 
A. guilinensis was once misidentified as a unique genotype of $A$. latifolia (Liang, 1988).

Although A. deliciosa var. chlorocarpa was recognized as a variety of $A$. deliciosa, its taxonomic status has been questioned (Li et al., 1996). Morphologically, A. deliciosa var. chlorocarpa is intermediate between $A$. deliciosa and A. chinensis for a majority of leaf and fruit characters (Li et al., 1996), but the pubescence on its fruits and stems is extremely similar to that of A. deliciosa var. deliciosa. The UPGMA phenogram obtained in this study suggests that it is closely related to A. deliciosa var. deliciosa. A cytogenetic study of $A$. deliciosa var. chlorocarpa indicated it was a tetraploid (He et al., 1998). A. chinensis has both diploids and tetraploids and has been considered a progenitor of hexaploid A. deliciosa (Cipriani et al., 1998; Huang et al., 1997; Testolin and Ferguson, 1997). A very close relationship between A. deliciosa var. deliciosa and var. chlorocarpa was also observed in isozyme analysis (Testolin and Ferguson, 1997). Based on the evidence available, A. deliciosa var. chlorocarpa has been speculated to be derived from hybridization of hexaploid var. deliciosa and diploid A. chinensis (He et al., 1998). In addition, the known overlapped geographical distribution of these three taxa also supports their close relationship (Li et al., 1996).

A. persicina is a recently published species (Huang and Wang, 1995). Based on their study, A. persicina appears to be closely related to $A$. zhejiangensis, which is in disagreement with a recent conclusion suggesting that it is closely related to A. grandiflora and $A$. hubeiensis based on leaf morphology (He et al., 2000). A. persicina and $A$. zhejiangensis formed a tight cluster at a genetic similarity about 0.60 , which is even higher than the average similarity between varieties (0.54). Plants of the two taxa, growing in WIBs repository, have only slight differences in the degrees of red color in their flowers and brown color in their anthers (Huang, personal observation). Reclassification of A. persicina is needed. Another related taxonomic uncertainty involves the positioning of $A$. rufa, a species native to Japan. A. rufa was once treated as a variety of $A$. arguta, in section Leiocarpae in Li's revision (1952). However, it was recently demonstrated to be more closely associated to A. hemsleyana by isozyme analysis (Testolin and Ferguson, 1997). Similarity between $A$. rufa and $A$. callosa var. henryi was also suggested based on flavonoid composition (Webby et al., 1994). The RAPD data provide additional support for positioning $A$. rufa in the group consisting of $A$. hemsleyana and A. zhejiangensis.

The low level of similarity observed within A. callos a reflects well its wide natural distribution and highly variable morphology (Li, 1952; Liang, 1984). In the phylogenetic analysis (Fig. 1), A. callosa var. strigillosa was not clustered with its species group, but instead it closely clustered with $A$. hubeiensis and A. grandiflora, and adjacent to the A. chinensis /A. deliciosa complex. The possible hybrid origin of $A$. hubeiensis and $A$. grandiflora has been previously suggested based on their morphological similarity and sympatric distributions (Liang, 1984; Sun and Huang, 1994). The RAPD data suggest that A. callosa var. strigillosa could be a parent of the other taxa, or possibly that $A$. hubeiensis, A. grandiflora and A. callosa var. strigillosa all resulted from a single hybridization event between $A$. chinensis (or A. deliciosa) and $A$. callosa and after subsequent speciation processes it differentiated into the present taxa. The specific boundary of $A$. callosa needs to be reconsidered.

Of all the members of the Actinidia, A. fulvicoma is known to have the most named varieties and forms. Two varieties, $A$. fulvicoma var. fulvicoma and var. lanata, were examined in the present study, and found to be highly dissimilar. A. fulvicoma exhibits a wide range of morphological variation, and is considered to be a rather heterogeneous species. It is comprised of various forms and many diverse genotypes resulting from natural hybridization, and is still thought to be under rapid speciation (Liang, 1984). A recent phylogenetic analysis based on cpDNA revealed a very close relationship of A. fulvicoma with $A$. glaucophylla and A. cylindrica (Cipriani et al., 1998). In contrast, however, the RAPD data suggest that it is closely related to $A$. eriantha and $A$. styracifolia. Overlapping distributions and frequent hybridization among these taxa could contribute to their close relationship, and account for the high level of genetic heterogeneity observed within A. fulvicoma.

The taxonomic position of $A$. kolomikta has been controversial. It was first placed in the Ampulliferae by Dunn (1911) and later subsumed into the section Leiocarpae when Li (1952) revised the genus. However, recent evidence based on leaf flavonoids and isozymes indicated that it was quite distinct from any species in the section Leiocarpae (Testolin and Ferguson, 1997; Webby et al., 1994). Further evidence for this was provided by phylogenetic analyses based on cpDNA (Cipriani et al., 1998). In contrast to these reports, the RAPD data suggest moderate levels of similarity between A. kolomikta and other members in section Leiocarpae, and support retaining A. kolomikta in the section Leiocarpae.

Although there remain unresolved taxonomic relationships within the Actinidia, the phylogenetic relationships suggested by the RAPD data presented in this study supplement our current understanding of Actinidia taxonomy. A new revision of the intrageneric subdivisions of Actinidia based on geographic distribution seems to be a logical step forward if the apparent association between natural distribution and phylogenetic relationship is a result of different hybridization events. RAPD analysis is not usually considered a sufficient analyzing tool to obtain a robust phylogeny of an angiosperm genus such as Actinidia that probably has a large number of species derived from hybridization events, and single individuals in each taxon used in this study also imposes limitations on the validity of the conclusions. Additional studies are needed. These studies might attempt to integrate all of the available morphological and molecular data, or collect low copy gene sequence data, in an attempt to obtain further resolution within the genus. Nevertheless, the results presented in this study provide useful information to our ongoing efforts toward the conservation and germplasm management for kiwifruit.

\section{Literature Cited}

Ausubel, F.M., R. Brent, R.E. Kingston, D.D. Moore, J.G. Seidman, J.A. Smith, and K. Struhl. 1987. Current protocols in molecular biology. Wiley, New York.

Cipriani, G., R. Testolin, and R. Gardner. 1998. Restriction-size variation of PCR-amplified chloroplast DNA regions and its implication for the evolution and taxonomy of Actinidia. Theor. Appl. Genet. 96:389396.

Cui, Z-X. 1993. Actinidia in China. Shandong Scientific Press, Jinan, Shandong, China.

Dunn, S.T. 1911. A revision of the genus Actinidia Lindl. J. Linn. Soc. Lond. Bot. 39:394-410.

Ferguson, A.R. 1990a. The genus Actinidia, p. 15-35. In: I.J. Warrington and G.C. Weston (eds.). Kiwifruit: Science and management. Ray Richards Publ, Auckland, N.Z.

Ferguson, A.R. 1990b. Kiwifruit (Actinidia), p. 603-653. In: J.N. Moore and J.R. Ballington, Jr. (eds.). Genetic resources of temperate fruit and nut crops. vol. 2. Intl. Soc. Hort. Sci., The Netherlands. 
Ferguson, A.R. and E.G. Bollard. 1990. Domestication of the kiwifruit, p. 165-246. In: I.J. Warrington and G.C. Weston (eds.). Kiwifruit: Science and management. Ray Richards Publ., Auckland, N.Z.

Gilg, E. 1893. Dilleniaceae. In: A. Engler and K. Prantl (eds.). Die naturlichen Pflanzenfamilien.Verlag Wilhelm Engelmann, Lepzig.

He, Z-C., S-M. Wang, H. Huang, H-Q. Huang. 1998. Study on chromosome numbers of six species and one variety in Actinidia Lindl. J. Wuhan Bot. Res. 16:299-301.

He, Z-C., Y. Zhung, H-T. Liu, X-H. Tang, L. Ye, D-S. Huang, and L-M. Xu. 2000. Quantitative taxonomic analyses of Actinidia (Actinidiaceae) in China based on micromorphological characters of foliar trichomes. Acta Phytotaxonomica Sinica 38:121-136.

Huang, H., F. Dane, Z-R. Wang, Z-W. Jiang, R-H. Huang, and S-M. Wang. 1997. Isozyme inheritance and variation in Actinidia. Heredity 78:328-336.

Huang, H., J-Q. Li, P. Lang, and S-M. Wang. 1999. Systematic relationships in Actinidia as revealed by cluster analysis of digitized morphological descriptors. Acta Hort. 498:71-78.

Huang, H., J-J. Gong, S-M. Wang, Z-C. He, Z-H. Zhang, and J-Q. Li. 2000. Genetic diversity in the genus Actinidia. Chinese Biodiversity $8: 1-12$.

Huang, H. and A.R. Ferguson. 2001. Kiwifruit in China. N.Z. J. Crop. Hort. Sci. 29:1-14

Huang, R-H. and S-M. Wang. 1995. Actinidia persicina-A new species of Actinidia. J. Wuhan Bot. Res. 13:113-115.

Jiang, H-Z. 1995. Two species of Actinidia from Yunnan. J. S.W. Agr. Univ. 17:93-96

Li, H-L. 1952. A taxonomic review of the genus Actinidia. J. Arnold Arbor. 33:1-61.

Li, J-Q., Q. Cai, and H. Huang. 2000. On the phylogeny of the genus Actinidia Lindley, p. 80-86. In: H. Huang (eds.). Advance in Actinidia research. Science Press, Beijing, China.

Li, R-G., M-Y. Liang, J-W. Li, and S-Z. Mao. 1996. Studies on the biological characteristic of genus Actinidia. Guihaia 16:265-272.

Liang, C-F. 1983. On the distribution of Actinidia. Guihaia 3:229-248.

Liang, C-F. 1984. Actinidia, p. 196-268. In: K-M. Feng (eds.). Flora reipublicae popularis sinicae. Science Press, Beijing, China.
Liang, C-F. 1988. New species Actinidia guilinensis. Guihaia 8:129_ 131.

McNeilage, M.A. and J.A. Considine. 1989. Chromosome studies in some Actinidia taxa and implications for breeding. N.Z. J. Bot. 27:7181.

Rohlf, F.J. 1994. NTSYS-pc, numerical taxonomy and multivariate analysis system. Exeter Publ., New York

Shi, S-D., Q-B. Wang, and Z-S. Zhang. 1994. New taxa of the genus Actinidia from Guizhou. Acta Bot. Yunnanica 16:335-347.

Sun, H-M. and R-H. Huang. 1994. A new species of Actinidia. J. Wuhan Bot. Res. 12:321-323

Testolin, R. and A.R. Ferguson. 1997. Isozyme polymorphism in the genus Actinidia and the origin of the kiwifruit genome. Syst. Bot. 22:685-700

Wagner, D.B., G.R. Furnier, M.A. Saghai-Maroof, S.M. Williams, B.P. Dancik, and R.W. Allard. 1987. Chloroplast DNA polymorphisms in lodgepole and jack pines and their hybrids. Proc. Natl. Acad. Sci. USA 84:2097-2100.

Webby, R.F., R.D. Wilson, and A.R. Ferguson. 1994. Leaf flavonoids of Actinidia. Biochem. Syst. Ecol. 22:277-286.

Williams, J.G.K, A.R. Kubelik, K.J. Livak, J.A. Rafalski, and S.V. Tingey. 1990. DNA polymorphisms amplified by arbitrary primers are useful as genetic markers. Nucl. Acid. Res. 18:6531-6535.

Yan, G., A.R. Ferguson, and M.A. McNeilage. 1994. Ploidy races in Actinidia chinensis: Implications for breeding. Euphytica 78:175-183.

Yan, G., J. Yao, A.R. Ferguson, M.A. McNeilage, A.G. Seal, and B.G. Murray. 1997. New reports of chromosomes numbers in Actinidia (Actinidiaceae). N.Z. J. Bot. 35:181-186.

Xiong,Z-T. and R-H. Huang. 1988. Chromosome numbers of 10 species and 3 varieties in Actinidia Lindl. Acta Phytotaxonomica Sinica 26:245-247

Xu, K-R. and D-Z Li. 1983. A preliminary research on the numerical classification of the genus Panax from China. Acta Phytotaxonomica Sinica 21:34-43

Zao, Y-L. and X-P. Liu. 1996. Study on the geographical distribution and floristic characteristics of the genus Actinidia Lindl. from Hunan. Guihaia 16:9-16 